\title{
Complex Web Construction: Additional Clues to Mechanical Properties-An Investigation by Middle School Students in Collaboration with MIT and JEOL, USA
}

W. Delise ${ }^{1}$, N. Lloyd ${ }^{1}$, J. Schmidt ${ }^{1}$, K. Baum ${ }^{1}$, D. Roos ${ }^{1}$ R. Dettelbach ${ }^{1}$, D. Maar ${ }^{1}$, V. Chandre ${ }^{1}$, K. Sanon $^{1}$, Z. Huseni ${ }^{1}$, D. Shattuck, Teacher ${ }^{1,2}$, Z. Qin ${ }^{2}$, M. Buehler ${ }^{2}$, V. Robertson ${ }^{3}$, and M. Shibata ${ }^{3}$

${ }^{1}$ Concord Middle School, Concord, MA, USA.

${ }^{2}$ Laboratory for Atomistic and Molecular Mechanics, Civil and Environmental Engineering Department, Massachusetts Institute of Technology, Cambridge, MA, USA.

${ }^{3}$ JEOL USA, Peabody, MA, USA.

Contact: Markus Buehler (mbuehler@MIT.EDU or 617.452.2750); Douglas Shattuck (shattuck@MIT.EDU or 978.318.1380)

Continuing, multi-year, collaboration among academia, industry, and public education has developed state-of-the-art classroom curricula and real research opportunities for students that were heretofore unavailable in public schools.

Collaborative outreach among academic institutions, and vendors of advanced instruments can offer substantial benefit to public schools. Presented here in poster format are the preliminary results of a continuing investigation conducted by ten middle school students into the mechanical properties of spider webs being studied at MIT's Laboratory for Atomistic and Molecular Mechanics (LAMM). Students began with images and technology provided by JEOL, USA for MIT and this program was conducted as part of ongoing cooperative outreach among MIT DCEE, LAMM, JEOL USA, and Concord Middle School.

This poster begins with a photo of naturally occurring web of a well-fed Orb Weaver (frame 1) that consists of rays emanating from the center that attach the web to its support structure. These lines are in tension and support the dead, live, and environmental loads. Chords or cross threads link the rays to form a familiar net-like web. An optical micrograph (frame 2) presents a web segment made by a "sheet web" producing spider of the Araneidae family harvested at MIT. Micrographic enhancements, provided by JEOL, (frames 3, 4, and 5) reveal a unique pattern in which rays of web are connected by chords that helically wrap each ray. At 1000x the complexity of junctions between ray and chord is revealed.

The goal of the investigation was to determine whether the helically wrapped chords in frames 3 , 4, and 5 provided any mechanical advantage when it comes to managing normal tensile forces acting on the web. The coiling structure at the junction is geometrically so complex that it could not be printed by a 3D printer and studied as had been done by the collaborators [1], [2]. This offers good motivation to conduct the investigation with the current strategy.

Students evaluated several materials and selected polyester sewing thread, monofilament fishing line, and packaging cord, to serve as analogues for spider silk. Prototype webs were hand woven (frame 6) along with other means for comparison. The design allows for weaving the webs so as to stress them along the radial (Y) axis, the chord (X) axis, and across (normal to) the X-Y plane [Z-axis]. Horizontal force tables and other apparatus were assembled to support the investigation.

Preliminary results indicate the Araneidae design may not support any more weight than the "Control" weave but that the design may have the ability to mechanically absorb force in conjunction with the elastic properties of the silk (Frames $7 \& 8$ ). The data suggest that the spider's weave distributes the load 
over several threads and may enhance the load bearing capacity in the X, Y, and Z directions (Frame 9). The weave also appears to provide a level of resilience to the web that is greater than that of the control (Frame 8). Moreover, we feel our data are consistent with findings of other researchers in the Laboratory and elsewhere but warrant further study [3], [4], [5], [6]. Future investigations may focus on enhancing robustness of the apparatus and precision of the protocol.

In addition to reinforcing concepts developed during research, this activity dramatically demonstrates the links among public school learning, academic research, and the private sector that supports them [7].

\section{References:}

[1] Zhao Qin, et al "Structural optimization of 3D-printed synthetic spider webs for high strength", Nature Communications, 2015

[2] Zhao Qin, Markus J. Buehler "Spider silk: Webs measure up", Nature materials, 2013

[3] S.W. Cranford, et al, "Nonlinear material behaviour of spider silk yields robust webs," Nature, Vol. 482, pp. 72-76, 2012

[4] N. Pugno, S. Cranford, M.J. Buehler, "Synergetic material and structure optimization yields strong and robust spider web anchorages," Small, Vol. 9(16), pp. 2747-2756, 2013

[5] Anna Tarakanova, Markus J. Buehler "The role of capture spiral silk properties in the diversification of orb webs", J. R. Soc. Interface, 2012

[6] Koebley, S.R., F.Volrath, and C. Schneipp, “Toughness-enhancing metastructure in the recluse spider's looped ribbon silk.”, J. R. Soc. Materials Horizons, 2017

[7] The student authors acknowledge and thank the Laboratory for Atomistic and Molecular Mechanics at MIT and the educational outreach of JEOL, USA.

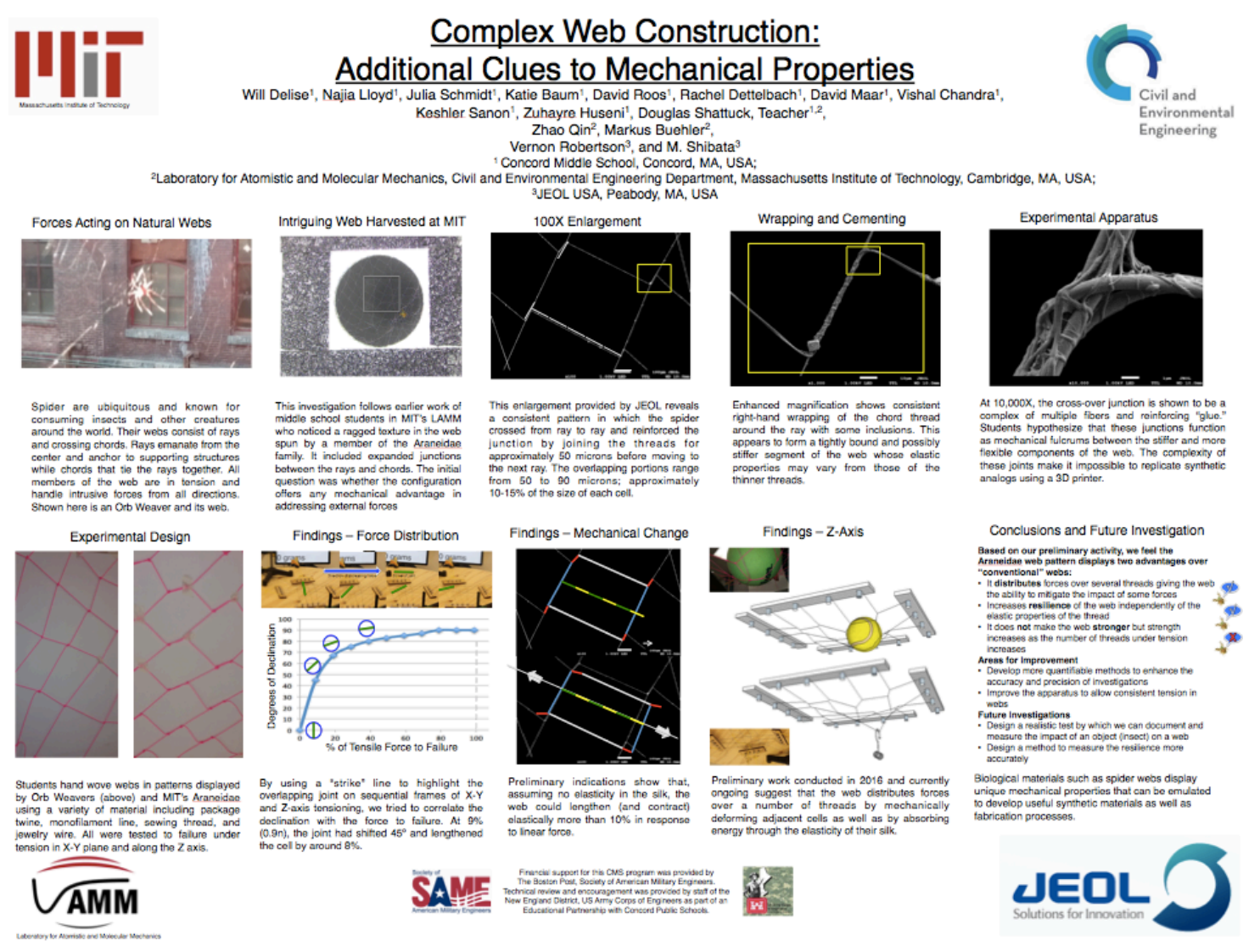

